\title{
COMPARACIÓN ENTRE LA PROPUESTA GENERADAS POR EL MODELO DE SIMULACION DE RIEGO OPTIMIZADO CON UN RIEGO CONSTANTE EN CULTIVO DE ZANAHORIA EN BRASIL
}

\author{
Conceição, B.C.L. ${ }^{1}$,Carvalhoa, D.F. ${ }^{2}$, Domínguez, A. ${ }^{3}$, Tarjuelo, J.M. ${ }^{4}$,Martínez-Romero,A. ${ }^{5}$ \\ ${ }^{1}$ Ingeniero Agrónomo, Investigador; ${ }^{2}$ Dr. Ingeniero Agrícola, Profesor, Departamento de \\ Ingeniería, Universidad Federal de Rio de Janeiro, BR 465, km 7, 23890-000 Seropédica-RJ \\ (Brasil) Barrow, carvalho@ufrrj.br; \\ ${ }^{3}$ Dr. Ingeniero Agrónomo, Profesor Contratado Doctor; ${ }^{4}$ Dr. Ingeniero Agrónomo, \\ Catedrático de Universidad. ${ }^{5}$ Dr. Ingeniero Agrónomo, Investigador; Centro Regional de \\ Estudios del Agua (CREA), Universidad de Castilla-La Mancha, Ctra. de Las Peñas km. 3.2, \\ 02071 Albacete, brunojlellismaria@yahoo.com.br; alfonso.dominguez@uclm.es; \\ jose.tarjuelo@uclm.es; angel.mromero@uclm.es;
}

\section{Resumen}

Los modelos de simulación de cultivos son utilizados como herramientas de ayuda a la gestión de los recursos disponibles. Para el cultivo de la zanahoria, pocos trabajos han estudiado el efecto del riego deficitario sobre su rendimiento y la calidad de las cosechas. La metodología ORDI (optimized regulated deficit irrigation), integrada en el modelo MOPECO, optimiza la distribución del agua disponible para riego a lo largo del ciclo de un cultivo bajo condiciones de déficit. El estudio se realizó en un área experimental de SIPA - Integrado de Producción Agroecológica, que se encuentra en Seropédica-RJ, Brasil. El sistema de riego utilizado fue localizado por goteo, con un ramal por cada dos líneas de cultivo y un espaciamiento entre emisores de $10 \mathrm{~cm}$, aportando un caudal de 4 litros metro ${ }^{-1}$. Se evaluaron dos métodos de aplicación de riego deficitario, los llamados: CDI (nivel de déficit constante de agua) y ORDI (nivel de déficit variable optimizado por etapas). Para ambos métodos se aplicaron 6 relaciones ETa/ETm globales objetivo $(1,0,0,9,0,8,0,7,0,6$ y sin riego). En los dos años de ensayo no aparecieron diferencias estadísticamente significativas entre los rendimientos totales cuando se compararon las metodologías ORDI y CDI, para el mismo nivel de déficit. Sin embargo, un alto porcentaje de las raíces obtenidas con la estrategia CDI presentaron deficiencias en términos de calidad (malformaciones, tamaños inadecuados, grietas, etc) que ocasionaron una disminución del rendimiento comercial. En consecuencia, para un mismo volumen de agua de riego aplicado, la distribución del riego propuesta por ORDI fue más eficiente en términos de rendimiento comercial (llegando al $50 \%$ en el tratamiento 0,6 y de alrededor del $24 \%$ para el resto de tratamientos).

\section{1) Introducción.}

Actualmente, el uso eficiente y racional de los recursos hídricos es una prioridad del sector agrario (FAO, 2009). Los modelos de simulación de cultivos están siendo utilizados como herramientas de ayuda a la gestión de los recursos disponibles, mejorando la productividad con el consiguiente ahorro de costes (STEWART et al., 1977). Para el cultivo de la zanahoria, son pocos los trabajos que han estudiado el efecto del riego deficitario sobre su rendimiento y la calidad de las cosechas. La metodología ORDI (optimized regulated deficit irrigation) (Domínguez et al., 2012) optimiza la distribución del agua 
disponible para riego a lo largo del ciclo de un cultivo bajo condiciones de déficit. Esta metodología maximiza el rendimiento para un cierto nivel de déficit objetivo para todo el ciclo del cultivo (expresado como relación ETa/ETm global objetivo).

El objetivo de este trabajo fue comparar los rendimientos obtenidos por un cultivo de zanahoria en Brasil, sometido a 6 relaciones $\mathrm{ETa} / \mathrm{ETm}$ globales objetivo $(1,0,0,9,0,8,0,7$, 0,6 y sin riego) aplicando un riego deficitario constante por etapas "CDI" (la relación $\mathrm{ETa} / \mathrm{ETm}$ de cada etapa se mantuvo constante e igual a la objetivo) y un riego deficitario optimizado por etapas "ORDI" (la relación ETa/ETm de cada etapa es diferente, pero la global del ciclo es igual a la objetivo).

\section{2) Materiales y métodos}

El estudio se realizó en un área experimental de SIPA - Integrado de Producción Agroecológica, que se encuentra en Seropédica-RJ (latitud $22^{\circ} 48^{\prime} 00$ " S; longitud $43^{\circ} 41^{\prime} 00 " W$; altitud de $33 \mathrm{~m}$ ). El suelo de la zona se clasifica como Argisolo, el clima es Aw en la clasificación de Köppen. Las temperaturas medias anuales son aproximadamente de $28^{\circ} \mathrm{C}$ y la precipitación de unos $1200 \mathrm{~mm}$, con un período de 5 meses muy secos. Los experimentos de campo se realizaron del 7 agosto (siembra) al 8 noviembre (cosecha) en 2013 y del 13 de junio al 11 de septiembre en 2014. El cultivar de zanahoria utilizado fue Brasilia Irecê, que se caracteriza por desarrollar plantas de tamaño medio (de 25 a $35 \mathrm{~cm}$ ), sus raíces son cilíndricas con longitudes entre 15 y $22 \mathrm{~cm}$ y un diámetro de 3 a $4 \mathrm{~cm}$, es de color naranja claro y presenta baja incidencia a enfermedades como el hombro verde o púrpura. Además, esta variedad soporta bien el calor, es robusta y la tendencia a entrar en floración en condiciones de día largo es baja. El sistema de riego utilizado fue el localizado por goteo, con un ramal por cada dos líneas de cultivo y un espaciamiento de emisores de $10 \mathrm{~cm}$ aportando un caudal de 4 litros metro ${ }^{-1}$. Las dimensiones de las parcelas experimentales fueron $1,0 \mathrm{~m} \times 2,0 \mathrm{~m}$, donde las líneas de cultivo estaban separadas $0,25 \mathrm{~m}$. Los datos climáticos fueron registrados por una estación meteorológica situada a 20 metros de la zona de ensayo, las informaciones diarias fueran utilizadas para estimar la evapotranspiración de referencia pela ecuación de Penman-Montheih FAO56 (ALLEN et al., 1998).

Cada tratamiento consistió en cuatro parcelas (repeticiones), todos dentro del mismo sitio. Se evaluaron dos métodos de aplicación de riego deficitario, los llamados: CDI (déficit de agua constante) y ORDI (déficit de agua optimizado en etapas). Para ambos métodos se aplicaron 6 relaciones $\mathrm{ETa}$ /ETm globales objetivo $(1,0,0,9,0,8,0,7,0,6$ y sin riego). El riego fue aplicado cada dos días, programación que es utilizada convencionalmente por los productores de la zona que hacen uso del sistema de riego por goteo.

La metodología ORDI maximiza el rendimiento para un cierto nivel ETa/ETm objetivo global. Los datos de entrada al modelo son: el rendimiento potencial, la ETm acumulada para cada etapa de desarrollo del cultivo para un año de referencia, los valores de Ky (coeficiente de sensibilidad al estrés hídrico) de cada etapa de desarrollo que deben ser calibrados con anterioridad para la zona y la variedad, y la relación ETa/ETm global objetivo. Mediante un software de optimización no lineal, ORDI determina las relaciones ETa/ETm teóricas para cada etapa de Ky que maximiza el rendimiento del cultivo. Para calibrar y validar los coeficientes de Ky se utilizaron los datos de dos ensayos realizados en Brasil en los años 2010 y 2011 (Carvalho et al., 2014). Las relaciones ETa/ETm determinadas por ORDI para cada etapa de desarrollo (establecimiento, desarrollo vegetativo, etapa media y maduración), en función de la ETa/ETm global objetivo fueron: 
- $\quad 1^{\text {a }}$ campaña 2013: $E T a / E T m$ objetivo $=0,6(0,95,0,63,0,50$ y 0,50$), E T a / E T m$ objetivo $=0,7(1,0,0,96,0,56$ y 0,50$), E T a / E T m$ objetivo $=0,8 \quad(1,0,1,0,0,78$ y $0,50)$, y ETa/ETm objetivo $=0,9(1,0,1,0,0,98$ y 0,59$)$.

- $\quad 2^{\text {a }}$ campaña 2014: ETa/ETm objetivo $=0,6(0,95,0,61,0,50$ y 0,50$), E T a / E T m$ objetivo $=0,7(1,0,0,94,0,54$ y 0,50$), E T a / E T m$ objetivo $=0,8(1,0,1,00,77$ y $0,50)$ y $\mathrm{ETa} / \mathrm{ETm}$ objetivo $=0,9(1,0,1,0,0,98$ y 0,58$)$.

Obviamente, las relaciones $\mathrm{ETa} / \mathrm{ETm}$ objetivo $=1 \mathrm{y}$ sin riego no pueden optimizarse. En el caso de los tratamientos CDI la relación ETa/ETm de cada etapa se mantuvo constante e igual a la objetivo en todas las etapas.

Este estudio evaluó la longitud de la raíz, la productividad total y la productividad comercial, además de la aparición de daños y el ataque de nematodos. Tras la cosecha, las raíces se lavaron y se clasificaron en cinco niveles comerciales: $10(>10<14 \mathrm{~cm}), 14(>14$ $<18 \mathrm{~cm}), 18(>18<22 \mathrm{~cm}), 22(>22<26 \mathrm{~cm})$ y $26(>26 \mathrm{~cm}) ;$ y no comercial $(\leq 10 \mathrm{~cm}$ y $/$ o defectuosa) (HortiBrasil, 2002).

\section{3) Resultados y discusión}

En los dos años de ensayo no aparecieron diferencias estadísticamente significativas entre los rendimientos totales cuando se compararon las metodologías ORDI y CDI. Sin embargo, un alto porcentaje de las raíces obtenidas con la estrategia CDI presentaron deficiencias en términos de calidad (malformaciones, tamaños inadecuados, grietas, etc) que ocasionaron una disminución del rendimiento comercial. En este sentido, los rendimientos comerciales de los tratamientos ORDI fueron significativamente superiores a los CDI, llegando al $50 \%$ en el tratamiento 0,6 y de alrededor del $24 \%$ para el resto de tratamientos (Tabla 1).

Tabla 1. Rendimiento total y comercial obtenido por los tratamientos CDI y ORDI.

\begin{tabular}{|c|c|c|c|c|c|c|c|}
\hline & & & CDI & & & ORDI & \\
\hline & ETa/ETm & $\begin{array}{l}\text { Lámina } \\
(\mathbf{m m})\end{array}$ & $\begin{array}{l}\text { Rend. Total } \\
\left(\mathrm{t} \mathrm{ha}^{-1}\right)\end{array}$ & $\begin{array}{l}\text { Rend. } \\
\text { Comercial } \\
\left(\mathrm{t} \mathrm{ha}^{-1}\right)\end{array}$ & $\begin{array}{l}\text { Lámina } \\
(\mathrm{mm})\end{array}$ & $\begin{array}{l}\text { Rend. Total } \\
\left(\mathrm{t} \mathrm{ha}^{-1}\right)\end{array}$ & $\begin{array}{l}\text { Rend. } \\
\text { Comercial } \\
\left(\mathrm{t} \mathrm{ha}^{-1}\right)\end{array}$ \\
\hline & S/Irrig & $201,4^{*}$ & $29,21^{*}$ & $12,92^{*}$ & - & - & - \\
\hline & 0,6 & 285,0 & 39,68 & 19,84 & 276,7 & 37,92 & 28,44 \\
\hline & 0,7 & 298,9 & 47,62 & 28,91 & 295,6 & 46,92 & 37,28 \\
\hline రิ & 0,8 & 312,9 & 56,48 & 37,32 & 309,8 & 55,84 & 45,37 \\
\hline & 0,9 & 326,8 & 63,17 & 43,43 & 322,4 & 62,35 & 51,77 \\
\hline & 1,0 & $340,7^{*}$ & $66,70^{*}$ & $60,23^{*}$ & - & 政 & - \\
\hline & S/Irrig & $153,4^{*}$ & $39,10^{*}$ & $17,13^{*}$ & - & - & - \\
\hline & 0,6 & 224,3 & 55,53 & 29,66 & 216,33 & 53,36 & 44,77 \\
\hline 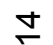 & 0,7 & 236,3 & 63,00 & 41,68 & 230,33 & 62,87 & 52,77 \\
\hline ำ & 0,8 & 247,9 & 68,20 & 47,50 & 247,77 & 68,48 & 60,99 \\
\hline & 0,9 & 259,7 & 71,77 & 55,53 & 263,71 & 73,28 & 66,98 \\
\hline & 1,0 & $271,5^{\star}$ & $77,25^{*}$ & $73,85^{*}$ & - & - & - \\
\hline
\end{tabular}

Como era de esperar, en ambos años los mayores rendimientos (total y comercial) fueron obtenidos por los tratamientos sin déficit. Estos coinciden con los obtenidos por otros autores. Nagaz et al. (2012) determinó que el rendimiento de zanahoria disminuyó con valores entre $80-60 \%$ de la ETa. Oliveira Neto (2013) y Gomes et al. (2012) obtuvieron en sus ensayos rendimientos máximos de aproximadamente $62 \mathrm{t} \mathrm{ha}{ }^{-1}$ y 75,90 $\mathrm{t} \mathrm{ha} \mathrm{ha}^{-1}$, 
respectivamente. Lopes et al. (2008) alcanzó un rendimiento de $39 \mathrm{t} \mathrm{ha}^{-1}$ en un tratamiento al que se aplicó un déficit del 0,6 ETm, similar a los resultados obtenidos en este estudio.

El desarrollo del cultivo está directamente relacionado con los valores de temperatura, ya que afectan a la duración de las etapas de desarrollo de la misma. Los promedios de temperatura de 2014 estuvieron dentro del rango óptimo para el desarrollo de la zanahoria $\left(18-25{ }^{\circ} \mathrm{C}\right)$, proporcionando una influencia positiva en el rendimiento de la cosecha. En relación a la productividad total de raíces de zanahoria en 2014, se observa un aumento de la productividad en todos los tratamientos comprados con el año 2013 y también un menor consumo de agua. Carvalho et al., (2014) encontró valores semejantes en dos ensayos realizados en 2010 y 2011 utilizando la misma variedad de zanahoria.

Los efectos de las láminas de riego sobe las diferentes metodologías para los dos años de cultivo (2013 y 2014), en relación al rendimiento total y al rendimiento comercial, se encuentran representados por las curvas de regresión y sus respectivos coeficientes de determinación obtenidos por el modelo polinomio cuadrático (Figuras 1 y 2 ).

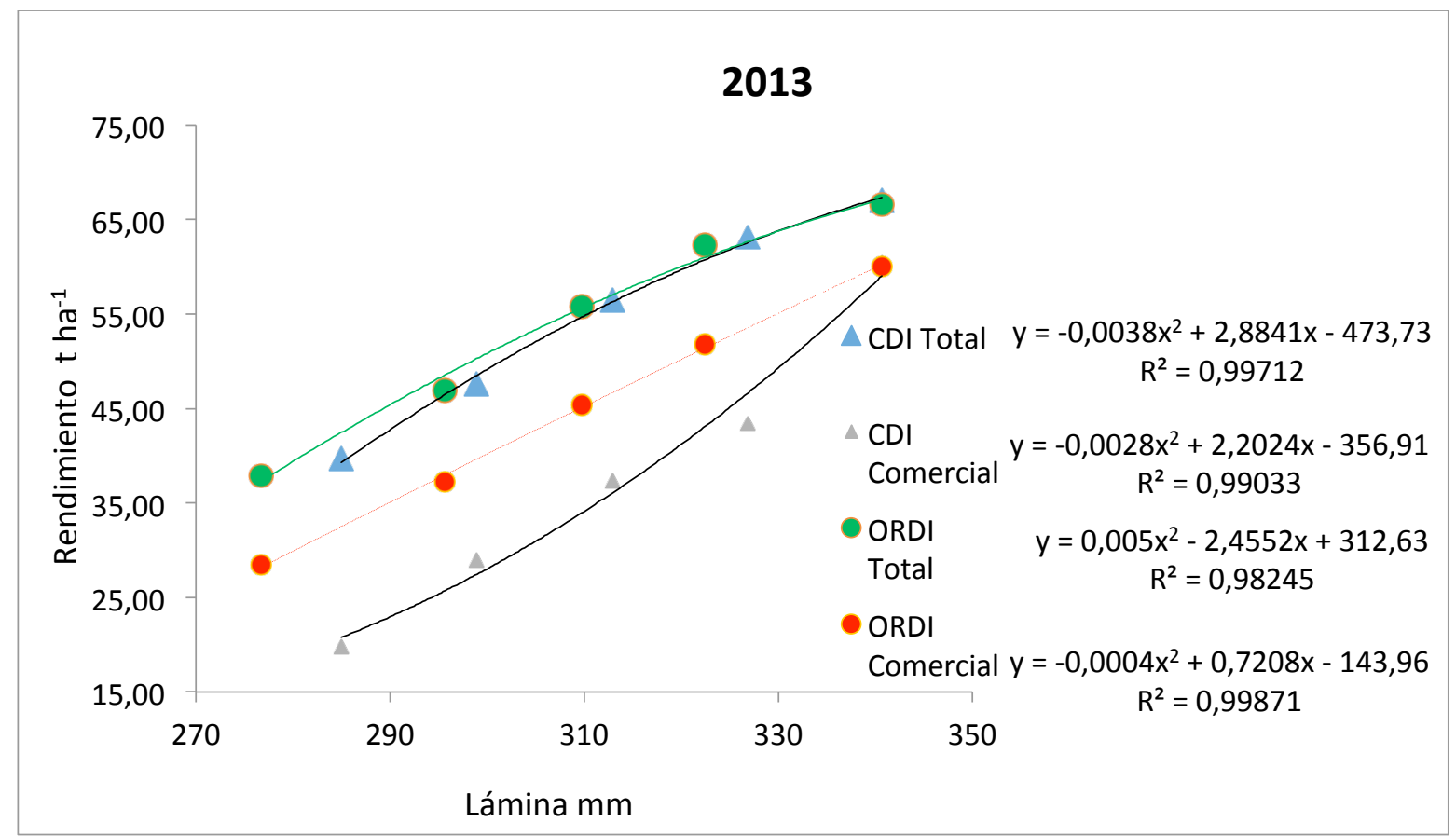

Figura 1. Curvas de respuesta para el rendimiento de raíces de Zanahoria para las metodologías CDI y ORDI en el cultivo del año de 2013. 


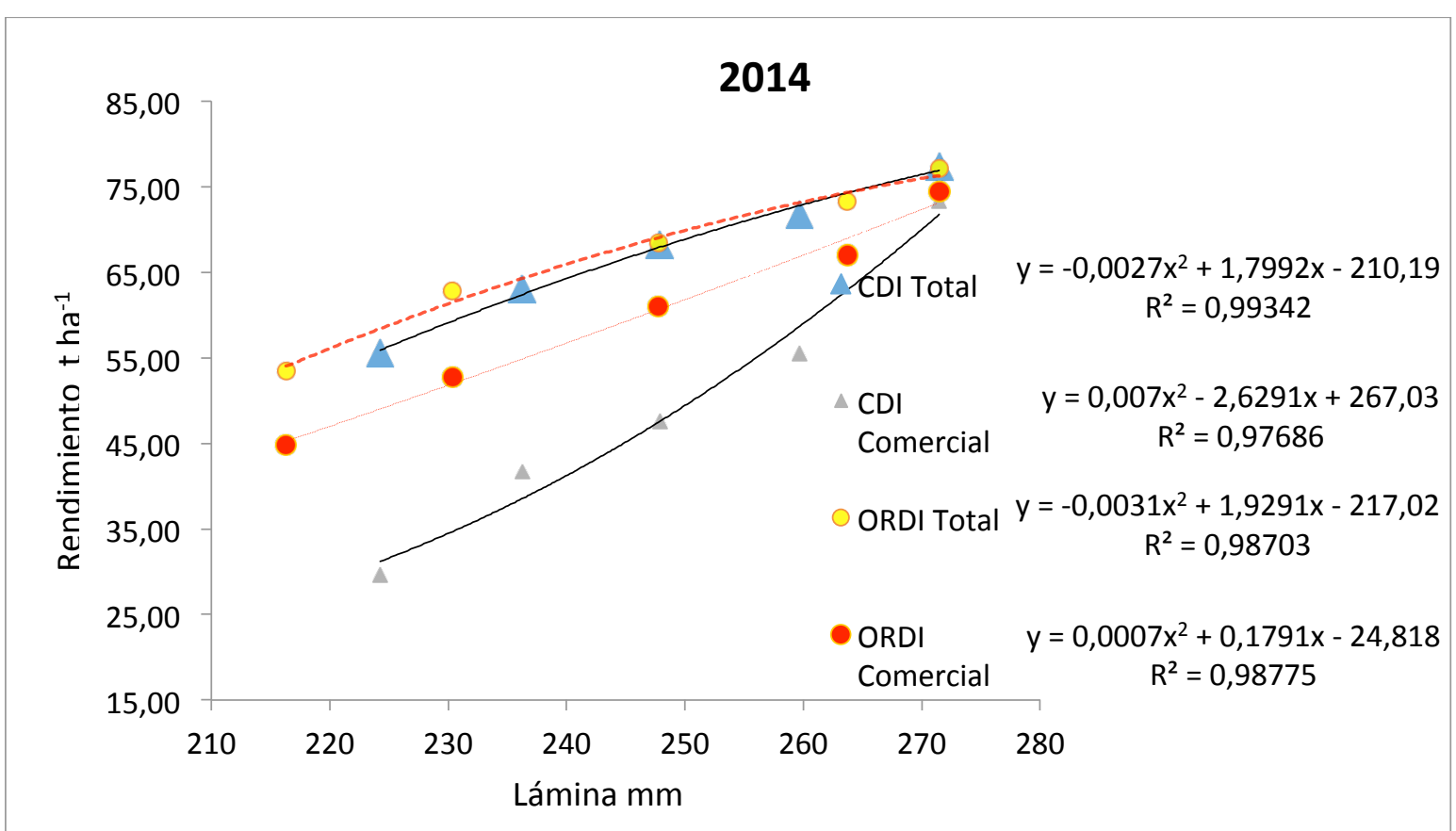

Figura 2. Curvas de respuesta para el rendimiento de raíces de Zanahoria para las metodologías CDI y ORDI en el cultivo del año de 2014.

\section{4) Conclusiones}

La metodología ORDI no ha logrado un mayor rendimiento total de raíces que la estrategia CDI. Sin embargo, en términos de rendimiento comercial, la estrategia ORDI ha sido mucho más eficiente. En consecuencia, en zonas con escasez de recursos hídricos donde se pretenda cultivar zanahoria bajo condiciones de riego deficitario, es conveniente aplicar las relaciones ETa/ETm propuestas por la metodología ORDI.

\section{5) Bibliografia}

Allen, R. G., Pereira, L. S., Raes, D., Smith, M. (1998). Crop evapotranspiration - Guidelines for computing crop water requirements. Rome: FAO, FAO Irrigation and Drainage. Paper 56. 319p.

Carvalho, D. F.; Neto, D. H. O.; Ribeiro, R. L. D.; Guerra, J. G. M.; Rouws, J. R. C. (2011). Manejo da irrigação associada a coberturas mortas vegetais no cultivo orgânico da beterraba. Engenharia Agrícola, v.31, p. 269-277

Carvalho D. F.; Domínguez A.; Oliveira Neto D. H.; Tarjuelo J. M.; Martínez-Romero A. (2014) Combination of date with déficit irrigation for improving the profitability of carrot in a tropical environment (Brasil). Scientia Horticulturae, v.179, p. 112-121..

Domínguez A., de Juan J.A., Tarjuelo J.M., Martínez R.S., Martínez-Romero A. (2012). Determination of optimal regulated deficit irrigation strategies for maize in a semi-arid environment. Agric. Water Manage, 110, 67-77.

Gomes D. P. (2012). Desempenho do consórcio de alface e cenoura, sob manejo orgânico com irrigação automatizada. Seropédica, RJ: UFRRJ, 64p. Tesis Maestria

HORTBRASIL- Instituto Brasileiro de Qualidade em Horticultura, 2002. Disponível em: http://hortibrasil.org.br/ Acessado em 13 de janeiro de 2013. 
Lopes, W. A. R.,; Negreiros, M. Z., Teófilo, T. M. S.; Alves, S. S. V., Martins,C. M., Nunes, G.H.S., Grangeiro, L.M.(2008).; Produtividade de cultivares de cenoura sob diferentes densidades de plantio. Revista Ceres. 483p

Nagaz, K.; Masmoud, M.M.; Mechlia.N. B. (2012). Impacts of irrigation regime with salina water on carret productivit and soil salinity. Journal of the Saudi Society of Agricultural Scienses. Tunisia. P. 19-27.

Oliveira Neto, D. H. (2013). Otimização do uso da água e produtividade da cenoura (Daucus carota) sob coberturas mortas no solo, em sistema agroecológico de produção. Seropédica, RJ: UFRRJ, 77p. Tesis de Doctorado.

Stewart, J. I., Hagan, R. M., Pruitt, W. O., Kanks, R. J., Riley, J. P., Danilson, R. E., Franklin, W. T., Jackson, E. B. (1977). Optimizing crop production through control of water and salinity levels in the soil. Reports. Paper 67 